\title{
Platelets: Pathophysiological Relevance and Therapeutic Potential
}

\author{
Hara Prasad Pati ${ }^{1} \cdot$ Prashant Sharma $^{2}$
}

Received: 25 April 2017/ Accepted: 25 April 2017/Published online: 28 April 2017

(C) Indian Society of Haematology \& Transfusion Medicine 2017

Platelets play key roles in physiological and pathological haemostasis, inflammation, wound healing and immune responses. This issue of the IJHBT contains several articles on the relevance of these tiny, enucleate, discoid cellular fragments across different clinical settings.

Among the quantitative platelet disorders, immune thrombocytopenia (ITP) of childhood is the most prevalent. Most children with acuteITP undergo rapid resolution. However, chronic ITP, i.e. platelet counts less than $100 \times 10^{9} / \mathrm{L}$ persisting for over 12 months, persists in nearly $10-20 \%$ patients. Clinical risk factors for chronicity, elegantly delineated in a recent meta-analysis [1], include older age, female gender, a lack of preceding infection or vaccination, gradual onset of symptoms and the use of a therapeutic regimen combining intravenous immunoglobulin (IVIg) with standard-dose methyl prednisolone. The last is especially interesting since IVIg alone was found to confer a protective effect, possibly due to its ability to restore regulatory T-cell numbers in responsive patients [1].

Chronic ITP possibly develops more commonly in children with less profound thrombocytopenia at diagnosis, higher mean platelet volumes and lower total leukocyte and lymphocyte counts. Serological studies show that the presence of anti-nuclear antibodies increases risk while the presence of platelet-associated IgM and anti-glycoprotein antibodies lowers it [1]. Genetic factorshave been analysed by several studies, with almost uniformly disappointing results. Only $T G F \beta 1 \mathrm{~cd} 25$ allele A and ILA intron 3 allele RP1 were shown to correlate consistently with the risk of

Hara Prasad Pati

harappati@yahoo.co.in

1 New Delhi, India

2 Chandigarh, India development of chronic ITP [2, 3]. The need for new and accurate biomarkers of prognosis is therefore real and urgent.

In this context, the study by Saeidi et al. in this issue [4] represents yet another attempt to utilize a genetic marker to predict treatment response. They quantitated $C X C R 4$ gene mRNA in 35 acute ITP patients using real-time PCR, and found that while levels differed between patients-versuscontrols as well as among patients, they did not correlate with the 1-week responses to first-line ITP therapies. However, what is noteworthy that they found CXCR4 levels to uniformly decline after treatment in acute ITP, whereas two prior papers had found CXCR4 levels to increase in chronic ITP [5, 6]. This hints that the marker may have potential value in predicting chronicity.A follow-up of their cohort would be clearly very interesting. Another intriguing aspect of their paper is that 18 out of 35 of their patients were born of consanguineous marriages and two had a family history of ITP. The cDNA of this Iranian pediatric patient group could therefore be invaluable in future high-throughput studies to identify genetic lesions that might modulate risk of developing acute ITP.

In the other papers on platelets, two studies deal with the influence of platelet mass components (typically defined as platelet count multiplied by the mean platelet volume, MPV) on a neoplastic and an infectious disorder. In the first of these, Ayer et al. [7] from Turkey studied the roles of MPV and JAK2 mutational status on thromboembolic events in essential thrombocythemia (ET) and polycythemia vera (PV) patients. While MPV was marginally lower in ET than in PV and in controls, it had no significant influence on thromboembolic events. This result is in line with recent opinion that advises caution in extrapolating physical parameters like MPV, plateletcrit and platelet distribution width as surrogates for activation and functional status of platelets [8]. 
The other paper by Sridhar et al. [9] looks at contributions of various parameters including clinical findings, degree of thrombocytopenia and D-dimer levels in predicting the risk of early disseminated intravascular coagumation (DIC) and thus bleeding in 60 children with dengue fever. Their conclusion, that clinical features of shock need to be factored in over and above the platelet count and D-dimer level, underscores the lesson that DIC is a clinicpathological diagnosis and the choice and intensity of component therapy needs to be guided by several indicators rather than just one or two lab parameters.

And finally, Arora et al. [10] have reviewed plateletderived biomaterials in this issue. Biomaterials are substances used in medical therapeutic or diagnostic procedures that have been processed or engineered to interact in vivo or in vitro with components of living systems. Examples of their use include fibrin glue or sealants to attain surgical hemostasis, platelet gels to promote wound healing and bone regeneration and platelet lysates with applications in ex vivo stem cell expansion. The production and marketing of biomaterials on an industrial scale entails stringent good laboratory practices as well as regulatory and quality control issues in addition to the robust evaluation of their clinical efficiency. The review touches upon all of these aspects.

In summary, the intrigues and the controversies around platelets as well as the diagnostic and therapeutic opportunities they promise truly cement their status as small cells with large impacts.

\section{References}

1. Heitink-Pollé KM, Nijsten J, Boonacker CW, de Haas M, Bruin MC (2014) Clinical and laboratory predictors of chronic immune thrombocytopenia in children: a systematic review and metaanalysis. Blood 124:3295-3307

2. Wu KH, Peng CT, Li TC, Wan L, Tsai CH, Lan SJ, Chang MC, Tsai FJ (2005) Interleukin 4, interleukin 6 and interleukin 10 polymorphisms in children with acute and chronic immune thrombocytopenic purpura. Br J Haematol 128:849-852

3. Atabay B, Oren H, Irken G, Kizildağ S, Tunali S, Türker M, Yilmaz S (2003) Role of transforming growth factor-beta 1 gene polymorphisms in childhood idiopathic thrombocytopenic purpura. J Pediatr Hematol Oncol 25:885-889

4. Saeidi S, Mohammadi-Asl J, Far MAJ, Asnafi AA, Dehuri F, Tavakolifar Y, SakiIsN (2016) There a relationship between CXCR4 gene expression and prognosis of immune thrombocytopenia in children? Indian J Hematol Blood Transfus 1-6. doi:10.1007/s12288-016-0648-0

5. Wang J-D, Ou T-T, Wang C-J, Chang T-K, Lee H-J (2010) Platelet apoptosis resistance and increased CXCR4 expression in pediatric patients with chronic immune thrombocytopenic purpura. Thromb Res 126(4):311-318

6. Olsson B, Ridell B, Carlsson L, Jacobsson S, Wadenvik H (2008) Recruitment of T cells into bone marrow of ITP patients possibly due to elevated expression of VLA-4 and CX3CR1. Blood 112(4):1078-1084

7. Ayer M, Menken İ, Yamak M, Ayer FA, Kırkızlar O, Aktuğlu MB (2016) The impact of mean platelet volume (MPV) and JAK2 mutation on thrombosis in chronic myeloproliferative diseases. Indian J Hematol Blood Transfus 1-7. doi:10.1007/s12288-0160685-8

8. Beyan C, Beyan E (2017) Mean platelet volume may not be an alternative test instead of platelet aggregation in patients with venous thromboembolism. Thromb Res 153:50

9. Sridhar A, Sunil Kumar BM, Rau A, Rau ATK (2016) A correlation of the platelet count with D-dimer levels as an indicator for component therapy in children with dengue hemorrhagic fever. Indian J Hematol Blood Transfus 1-6. doi:10.1007/s12288-0160686-7

10. Arora S, Agnihotri N (2016) Platelet derived biomaterials for therapeutic use: review of technical aspects. Indian J Hematol Blood Transfus 1-9. doi:10.1007/s12288-016-0669-8 